\title{
Finding the Dimension Which Minimize Material Cost of Industrial Plastic Products in Ethiopia
}

\author{
Muluken Admasu* \\ Mathematics Stream, Kombolcha Technology Institute, Wollo University \\ PO box 208, Kombolcha, Ethiopia \\ Ashager Adane \\ Mathematics Stream, Kombolcha Technology Institute, Wollo University \\ PO box 208, Kombolcha, Ethiopia
}

\begin{abstract}
The purpose of this study focused on identifying and verifying an effective and reliable dimension of plastic products for minimize the material cost. As the costs of manufacturing product decrease, the way that consumers interact with producers may change. To specify the best optimal dimension, two criteria's, that has been done using numerical and graphical techniques, were in order to compare and contracts the cost of material. During estimation of non-linear system of equations MATLAB (R2015a) software are used. In many instances, the cost of producing a product using traditional methods exceeds that of mathematical optimization methods. The material cost difference between mathematical optimization and traditional method was presented graphically.
\end{abstract}

Keywords: Mathematical cost optimization, material cost difference, products dimension

DOI: $10.7176 / \mathrm{JNSR} / 11-13-03$

Publication date:July $31^{\text {st }} 2020$

\section{Introduction}

Modern design and manufacturing depend heavily on the use of mathematics and software programs. Computer assisted design (CAD) harnesses the power of computers to speed up and improve the process of creating and design. In particular, the ability to modify an existing design drawing quickly it possible to examine more alternatives in an effort to find an optimum design. Mathematically the minimum cost dimension and shape of a product can be investigate. But, many survey studies indicate Ethiopian plastic industries still working traditionally. As the costs of manufacturing product decrease, this technology may change the way that consumers interact with producers. It can facilitate the customized production of strong light-weight products and it allows designs that were not possible with previous manufacturing techniques.

Plastic sheets in thicknesses up to $200 \mu \mathrm{m}$ are used to produce semi-rigid packaging such as pots, tubs and trays. The properties of plastic films and sheets are dependent on the plastic(s) used and the method of film manufacture together with any coating or lamination. There are many food applications for rigid and semi-rigid thermoformed containers. Examples include a wide range of dairy products, yoghurts etc. in single portion pots, fresh sandwich packs, compartmented trays to segregate assortments of chocolate confectionery and trays for biscuits.

Plastic has properties of strength and toughness and specific plastics can meet the needs of a wide temperature range, from deep-frozen food processing $\left(-40^{\circ} \mathrm{C}\right)$ and storage $\left(-20^{\circ} \mathrm{C}\right)$ to the high temperatures of retort sterilization $\left(121^{\circ} \mathrm{C}\right)$, and reheating of packaged food products by microwave $\left(100^{\circ} \mathrm{C}\right)$ and radiant heat $\left(200^{\circ} \mathrm{C}\right)$. The main reasons why plastics are used in food packaging are that they protect food from spoilage, can be integrated with food processing technology, do not interact with food, are relatively light in weight, are not prone to breakage, do not result in splintering and are available in a wide range of packaging structures, shapes and designs which present food products cost effectively, conveniently and attractively. During the period 2001--2013, local production of plastic packing materials has increased from 8,931 tons to 19,956 tons, registering an average annual growth rate of $8.44 \%$. During the recent five years (2009--2013) from the total local production of plastic packing materials the majority is accounted by rigid plastic packaging materials $(53.57 \%)$. The remaining $46.43 \%$ is accounted by flexible plastic packaging materials.

During the period 2001--2013 import of rigid plastic packing materials exhibits a significant growth. In 2002, import was only 73 tons and Birr 2.08 million in terms of volume and value, respectively. However, in 2013 import has increased to 7,293 tons and Birr 342.59 million in terms of volume and value respectively. During the recent five years (2009--2013), in terms of volume, from the total import of rigid plastic packing materials the great majority (on average 75.74\%) is accounted by bottle preforms, followed by boxes, cases, creates and similar articles (16.34\%), stoppers, lids, caps and other closures of plastic (4.97\%) and carboys, bottles, flasks and similar articles $(2.95 \%)$.

Total supply of flexible plastic packing materials which was 6,059 tons in 2001 has increased to 21,411 tons in 2013 , registering an average annual growth rate of $16.97 \%$. On average during the period under consideration 
(2001--2013) the share of local production was $46.70 \%$, which implies that the great majority $(53.30 \%)$ of the local demand for flexible plastic packing materials is met through import. Total supply or apparent consumption of rigid plastic packing materials exhibits a year to year growth. The yearly average apparent consumption, which was about 4,600 tons during the period 2001--2003, has increased to a yearly average of about 17,300 tons during the period 2012--2013. During the period under consideration (2001--2013) total supply of rigid plastic packing material has registered an average annual growth rate of $12.79 \%$. Apparent consumption of laminated plastic packing materials has increased from a yearly average of 106 tons in the year 2001--2006 to a yearly average of 423 tons during the period 2011--2013, with a yearly average growth rate of $26.62 \%$

The local demand for flexible plastic packing materials is projected to increase from 24,562 tons in 2015 to 49,430 tons and 99,448 tons by the years 2020 and 2025 respectively. Moreover, by year 2030 the demand is projected to reach 200,052 tons. The local demand for rigid plastic packing materials is projected to increase from 19,077 tons in 2015 to 41,127 tons and 85,479 tons by the years 2020 and 2025 respectively. Moreover, by year 2030 the demand is projected to reach 174,686 tons. Similarly, the local demand for laminated plastic packing materials projected to increase from 594 tons in 2015 to 1,316 tons, 2,768 tons and 5,690 tons by the years 2020 , 2025 and 2030, respectively.

\section{Objective of the Study}

The objective of this paper is to present the minimum cost of dimension of different industrial plastic products and to show the difference cost of material between selections of dimension using mathematical optimization method and traditional methods in Ethiopia.

\section{Literature Review}

Studies are being done upon minimum cost of product in order to reflect on economic growth, employment, savings and environment, conservation of assets, investments and environmental impacts. In order to get minimum curvature variation new technology curve and surface design that combines a geometrically based specification with constrained optimization was developed [Henry, 1992]. The complex shapes with tight tolerances which are increasingly adopted in industrial applications make it become a great challenge for geometrical product specification to control the geometrical variations along the product lifecycle. Significant efforts are being devoted to develop systems that can support geometrical product specifications. Among them, Geo Spelling [Ballu, 1995] [Mathieu, 2003] is a classical one, which has already standardized as ISO 17450-2005. The basic dimensions of potato tubers' shape were used for calculation of geometric mean diameter and volume by analyzing mathematical descriptive characteristics [A. Bubeníčková, 2011].

In Ethiopia, Central Statistical Authority (CSA) has been publishing results of the survey study of Manufacturing and electricity industries on annual basis since 1976 to provide users with reliable, compressive and timely statistical data on these sector. DAB development and research and training PLC (2014) studied Survey of Ethiopian Manufacturing Sector. On this study most products produced by the manufacturing subsectors consumed by domestic users. Almost half of the repliers reported that they do not have any export destinations for their products. African countries are the major export destinations, which got about $26 \%$ of the interviewed industries reply followed by North America (11.2\%), Western Europe (10.7\%) and Asia (8\%) while other areas had very insignificant share. Inadequate and poor quality imported raw materials and technologies, along with low level of technical skills become major challenge.

The Embassy of Japan in Ethiopia (2018) conduct Series of Studies on different Industries in Ethiopia and show that using traditional method and huge amount of wastage material become major challenge to compute international market. Tekeba Eshetie (2018) studied Ethiopia's Manufacturing Industry Opportunities, Challenges and Way Forward. On this study one of the challenge of industries were using traditional selection of dimension of industrial products. Furthermore, the researcher indicate limited research on manufacturing industries including end market study is a great challenge. Because of this paper initiation and observing the problem in industries and vocational college's teachers understanding we try to show scientifically the gap of end market about traditional ways of choosing dimensions.

\section{Material and Methods}

The study used previous data obtained from the Ministry of Industry (MoI) and Central statistics agency (CSA) in Ethiopia. We consider 40 different products which are mostly manufactured based on their size and thickness and the data is taken from 2018/19 industries annual report. Our analysis approach is to determine only material costs that can show easily how much industries loss because of their choice of dimension traditionally. The mathematical optimization methods material cost and traditional method material cost was calculated. The non-linear equation and non-linear system of equations were done in a windows machine installed with MATLAB version (R2015a), to analyze cost of material by using excel functions and results were presented in terms of numerals, tables and graphs. 


\section{Mathematical Analysis on Optimization the cost of products}

Manufacturing is critical and is probably the most important engine of long-term growth and development. As countries transform from primary agricultural-based economies to manufacturing based ones, more sustainable revenue for growth is obtained. Including Ballu and Tekeba most researchers recommended strongly optimize the cost and dimension of product is crucial for sustainable development and market computation.

In Ethiopia, most storage containers are cylindrical, rectangular and frustum of conical shape. But for special purpose hemispherical on the top of cylinder is also mostly manufactured. Each of these shapes has its benefits and its limitations, and the one you choose depends on two main considerations - the amount of space you have available for storing these containers, and your personal preferences and tastes. Products are manufactured the same thickness and different thickness material for different purpose. The cost of material in this paper expressed in Ethiopian money called Ethiopian Birr (ETB). Based on Ethiopian manufacturing survey study the average cost material is 500-800ETB per square meter depends on the thickness of material. Mostly for bottom is 800 ETB per square meter, for side 500 ETB per square meter and for top is 600 ETB per square meter. If the material type and thickness is the same for all sides of product reducing the total surface area is reducing cost. But it doesn't means for different types of material usage.

Science and technological change occurs through the process of innovation, invention, and diffusion that leads to the transformation of ideas and knowledge into tangible products that have highly utility to human needs. As science and technology advances, the systems of production become capital intensive, labor saving and cost effective.

\subsection{Optimization of Cylindrical Shape}

Since, our study focused on identifying and quantifying the loss of cost associated with the selection of dimension of products then the cost of material using the same material and sticking fragments is

$$
\begin{gathered}
C=2 \pi r(0.05)(r+h)=0.1 \pi r\left(r+\frac{V}{\pi r^{2}}\right)=0.1 \pi\left(r^{2}+\frac{V}{\pi r}\right) \\
C^{\prime}(r)=0.1 \pi\left(2 r-\frac{\pi V}{(\pi r)^{2}}\right)=0
\end{gathered}
$$

The critical values are $r=0$ and $r=\sqrt[3]{\frac{V}{2 \pi}}$, to determine the only real critical value weather it is local maximum or minimum value use second derivative test.

$$
\mathrm{C}^{\prime \prime}(\mathrm{r})=0.2 \pi\left(1+\frac{\mathrm{V}}{\pi \mathrm{r}^{3}}\right)>0
$$

Thus, by second derivative test the minimum cost of cylindrical shape product is

Where the dimension of cylindrical shape is

$$
\mathrm{C}=\frac{2}{15} \sqrt[3]{\pi \mathrm{V}^{2}}
$$

$$
r=\sqrt[3]{\frac{\mathrm{V}}{2 \pi}} \text { and } \mathrm{h}=\sqrt[3]{\frac{4 \mathrm{~V}}{\pi}}
$$

The cost of material using the same material and folding fragments is

$$
\begin{gathered}
C=2 \pi r(0.05)(r+h)+2 \pi r(1.25)(0.05)=0.1 \pi\left(r^{2}+\frac{v}{\pi r}\right)+0.125 \pi r \\
C^{\prime}(r)=0.1 \pi\left(2 r-\frac{\pi V}{(\pi r)^{2}}+1.25\right)
\end{gathered}
$$

The critical value will exist on the root of equation

$$
2 \pi r^{3}+1.25 \pi r^{2}-V=0
$$

Equation (1.3) can be solve using MATLAB software and we have non-zero critical value $r=4.1027$ for $V=$ $500, r=5.2187$ for $V=1000, r=6.6257$ for $V=2000, r=7.613$ for $V=3000$ and $r=9.0631$ for $V=$ 5000

Since $C^{\prime \prime}(r)>0$ for all above values of $r$ and $V$ then by second derivative test the cost of material equation (1.2) is minimum.

The cost of material using different material and sticking fragments is

$$
\begin{gathered}
C=\pi r^{2}(0.08+0.06)+2 \pi r h(0.05)=0.14 \pi r^{2}+\frac{0.1 V}{r} \\
C^{\prime}(r)=0.28 \pi r-\frac{0.1 V}{r^{2}}=0
\end{gathered}
$$

We have non-zero critical value $r=\sqrt[3]{\frac{10 \mathrm{~V}}{28 \pi}}$

$$
\mathrm{C}^{\prime \prime}(\mathrm{r})=0.28 \pi+\frac{0.2 \mathrm{~V}}{\mathrm{r}^{3}}>0
$$


Thus, by second derivative test the minimum cost of cylindrical shape product is

Where the dimension of cylindrical shape is

$$
C=\left(\pi V^{2}\right)^{1 / 3}\left[\frac{7}{50} \pi\left(\frac{5}{14}\right)^{2 / 3}+\frac{1}{10}\left(\frac{14}{5}\right)^{1 / 3}\right]
$$

$$
r=\sqrt[3]{\frac{5 V}{14 \pi}} \text { and } h=\sqrt[3]{\frac{196 V}{25 \pi}}
$$

The cost of material using different material and folding fragments is

$$
\begin{gathered}
C=\pi r^{2}(0.08+0.06)+2 \pi r h(0.05)+2 \pi r(1.25)(0.06)=0.14 \pi r^{2}+\frac{0.1 V}{r}+0.15 \pi r \\
C^{\prime}(r)=0.28 \pi r-\frac{0.1 V}{r^{2}}+0.15 \pi=0
\end{gathered}
$$

The non-zero critical value will exist on the root of equation

$$
0.28 \pi r^{3}+0.15 \pi r^{2}-0.1 V=0
$$

Equation (1.6) can be solve using MATLAB software and we have non-zero critical value $r=3.6744$ for $V=500$, $r=4.6721$ for $V=1000, r=5.93$ for $V=20000, r=6.8126$ for $V=3000$ and $r=8.1089$ for $V=5000$. Since $C^{\prime \prime}(r)>0$ for all above values of $r$ and $V$ then by second derivative test the cost of material equation (1.5) is minimum.

\subsection{Optimization of Rectangular Shape}

For better optimization let us use square base instead of rectangular shape. The cost of material using the same material and sticking fragments is

We have non-zero critical value at $l=\sqrt[3]{V}$

$$
\begin{aligned}
C & =0.1\left(l^{2}+\frac{2 V}{l}\right) \\
C^{\prime}(l) & =0.1\left(2 l-\frac{2 V}{l^{2}}\right)=0
\end{aligned}
$$

$$
\begin{gathered}
C^{\prime \prime}(l)=0.1\left(2+\frac{4 V}{l^{3}}\right) \\
C^{\prime \prime}(\sqrt[3]{V})=0.6>0
\end{gathered}
$$

Thus, by second derivative test the minimum cost of rectangular shape product is

$$
C=0.1\left(V^{2 / 3}+2 V^{2 / 3}\right)=0.3 V^{2 / 3}
$$

Where, the dimension of rectangular shape $l=w=h=\sqrt[3]{V}$

The cost of material using the same material and folding fragments is

The non-zero critical value will exist on the root of equation

$$
\begin{aligned}
& C=0.1\left(l^{2}+\frac{2 V}{l}\right)+4 l(1.25)(0.05)=0.1\left(l^{2}+\frac{2 V}{l}\right)+0.25 l \\
& C^{\prime}(l)=0.1\left(2 l-\frac{2 V}{l^{2}}\right)+0.25 \\
& C^{\prime}(r)=0.28 \pi r-\frac{0.1 V}{r^{2}}+2.56 \pi=0
\end{aligned}
$$

Equation (1.9) can be solve using MATLAB software and we have non-zero critical value $l=7.5414$ for $V=$ $500, l=9.6002$ for $V=1000, l=12.196$ for $V=2000, l=14.0176$ for $V=3000$ and $l=16.6931$ for $V=$ 5000 . Since $C^{\prime \prime}(l)>0$ for all above values of $l$ and $V$ then by second derivative test the cost of material equation (1.8) is minimum.

The cost of material using different material and sticking fragments is

$$
\begin{gathered}
C=l^{2}(0.08)+4(0.05)(l h)+l^{2}(0.06)=0.14 l^{2}+\frac{0.2 \mathrm{~V}}{l} \\
C^{\prime}(l)=0.28 l-\frac{0.2 \mathrm{~V}}{l^{2}}=0
\end{gathered}
$$

We have non-zero critical value at $l=\sqrt[3]{\frac{5 V}{7}}$

$$
C^{\prime \prime}\left(\sqrt[3]{\frac{5 V}{7}}\right)=0.28+\frac{2.8 \mathrm{~V}}{5}>0
$$

Thus, by second derivative test the minimum cost of rectangular shape product is 


$$
C=0.14\left(\frac{5 V}{7}\right)^{2 / 3}+\frac{0.2 V}{\left(\frac{5 V}{7}\right)^{1 / 3}}=V^{2 / 3}\left[\frac{1}{10}\left(\frac{7}{5}\right)^{1 / 3}+\left(\frac{7}{625}\right)^{1 / 3}\right]
$$

Where the dimension of rectangular shape is $l=w=\sqrt[3]{\frac{5 V}{7}}$ and $h=\sqrt[3]{\frac{49 V}{25}}$

The cost of material using different material and folding fragments is

$$
\begin{gathered}
C=0.14 l^{2}+\frac{0.2 V}{l}+4 l(1.25)(0.06)=0.14 l^{2}+\frac{0.2 V}{l}+0.3 l \\
C^{\prime}(l)=0.28 l-\frac{0.2 V}{l^{2}}+0.3=0
\end{gathered}
$$

The non-zero critical value will exist on the root of equation

$$
2.8 l^{3}+3 l^{2}-2 V=0
$$

Equation (1.11) can be solve using MATLAB software and we have non-zero critical value $l=6.7552$ for $V=$ $500, l=8.5958$ for $V=1000, l=10.9164$ for $V=2000, l=12.5449$ for $V=3000$ and $l=14.9366$ for $V=5000$

Since $C^{\prime \prime}(l)>0$ for all above values of $r$ and $V$ then by second derivative test the cost of material equation (1.10) is minimum.

\subsection{Optimization of hemispherical on top of a cylindrical shape}

The volume of product is the volume sum of lower cylindrical shape and upper hemispherical shape. The cost of material using the same material and sticking fragments is

$$
\begin{gathered}
C=0.05\left(3 \pi r^{2}+2 \pi r h\right)=0.05\left(3 \pi r^{2}+2 \pi r \frac{V-\frac{2}{3} \pi r^{3}}{\pi r^{2}}\right)=0.05\left(3 \pi r^{2}+\frac{2 V}{r}-\frac{4}{3} \pi r^{2}\right) \\
=0.05\left(\frac{5}{3} \pi r^{2}+\frac{2 V}{r}\right) \\
C^{\prime}(r)=0.05\left(\frac{10}{3} \pi r-\frac{2 V}{r^{2}}\right)=0
\end{gathered}
$$

The non-zero critical value is $r=\sqrt[3]{\frac{3 V}{5 \pi}}$

Since, $C^{\prime \prime}(r)=0.1\left(\frac{5}{3} \pi+\frac{4 V}{r^{3}}\right)=0.1\left(\frac{5}{3} \pi+\frac{20}{3} \pi\right)=\frac{5}{6} \pi>0$ the by second derivative test $r=\sqrt[3]{\frac{3 V}{5 \pi}}$ is local minimum value.

The minimum cost of material using the same material and sticking fragments is

$$
C=0.05\left(\frac{5}{3} \pi r^{2}+\frac{2 V}{r}\right)=0.05\left(\frac{5}{3} \pi\left(\frac{3 V}{5 \pi}\right)^{2 / 3}+\frac{2 V}{\left(\frac{3 V}{5 \pi}\right)^{1 / 3}}\right)=\frac{3}{20}\left(\frac{5}{3} \pi V^{2}\right)^{1 / 3}
$$

Where, the dimension is $r=\sqrt[3]{\frac{3 V}{5 \pi}}$ and $h=\frac{V}{\pi r^{2}}-\frac{2}{3} r=\sqrt[3]{\frac{V}{\pi}}\left[\left(\frac{5}{3}\right)^{2 / 3}-\frac{2}{3}\left(\frac{3}{5}\right)^{1 / 3}\right]$

The cost of material using the same material and folding fragments is

$$
\begin{gathered}
C=0.05\left(\frac{5}{3} \pi r^{2}+\frac{2 V}{r}+2 \pi r(1.25)\right)=0.05\left(\frac{5}{3} \pi r^{2}+\frac{2 V}{r}+2.5 \pi r\right) \\
C^{\prime}(r)=0.1\left(\frac{5}{3} \pi r-\frac{V}{r^{2}}+1.25 \pi\right)=0
\end{gathered}
$$

The non-zero critical value will exist on the root of equation

$$
5 \pi r^{3}+3.75 \pi r^{2}-3 V=0
$$

Equation (1.13) can be solve using MATLAB software and we have non-zero critical value $r=8.063$ for $V=$ $3000, r=9.6037$ for $V=5000, r=12.162$ for $V=10000, r=13.957$ for $V=15000$ and $r=15.386$ for $V=20000$

Since $C^{\prime \prime}(r)>0$ for all above values of $r$ and $V$ then by second derivative test the cost of material equation (1.12) is minimum.

The cost of material using different material and sticking fragments is 


$$
\begin{gathered}
C=0.08 \pi r^{2}+2(0.06) \pi r^{2}+2 \pi r h(0.05)=0.2 \pi r^{2}+0.1 \pi r\left(\frac{V-\frac{2}{3} \pi r^{3}}{\pi r^{2}}\right)=0.2 \pi r^{2}+0.1\left(\frac{V}{r}-\frac{2}{3} \pi r^{2}\right) \\
=\frac{1}{5} \pi r^{2}+\frac{V}{10 r}-\frac{1}{15} \pi r^{2}=\frac{2}{15} \pi r^{2}+\frac{1}{10} \frac{V}{r} \\
C^{\prime}(r)=\frac{4}{15} \pi r-\frac{1}{10} \frac{V}{r^{2}}=0
\end{gathered}
$$

The non-zero critical value is $r=\sqrt[3]{\frac{3 V}{8 \pi}}$

Since, $C^{\prime \prime}\left(\sqrt[3]{\frac{3 V}{8 \pi}}\right)=\frac{4}{15} \pi+\frac{1}{5} \frac{V}{r^{3}}=\frac{4}{15} \pi+\pi=\frac{19}{15} \pi>0$ then by second derivative test $r=\sqrt[3]{\frac{3 V}{8 \pi}}$ is local minimum value.

The minimum cost of material using the different material and sticking fragments is

$$
C=\frac{2}{15} \pi\left(\frac{3 V}{8 \pi}\right)^{2 / 3}+\frac{1}{10} \frac{V}{\left(\frac{3 V}{8 \pi}\right)^{1 / 3}}=\sqrt[3]{\pi V^{2}}\left[\frac{2}{15}\left(\frac{3}{8}\right)^{2 / 3}+\frac{1}{10}\left(\frac{8}{3}\right)^{1 / 3}\right]
$$

Where, the dimension of the product is $r=\sqrt[3]{\frac{3 V}{8 \pi}}$ and $h=\left(\frac{V}{3 \pi}\right)^{1 / 3}\left[\left(\frac{V}{\pi}\right)^{1 / 3}-\frac{1}{2}\left(\frac{1}{3}\right)^{1 / 3}\right]$

The cost of material using different material and folding fragments is

$$
\begin{gathered}
C=\frac{2}{15} \pi r^{2}+\frac{1}{10} \frac{V}{r}+2 \pi r(1.25)(0.06)=\frac{2}{15} \pi r^{2}+\frac{1}{10} \frac{V}{r}+0.15 \pi r \\
C^{\prime}(r)=\frac{4}{15} \pi r-\frac{1}{10} \frac{V}{r^{2}}+\frac{15}{100} \pi=0
\end{gathered}
$$

The non-zero critical value will exist on the root of equation

$$
16 \pi r^{3}+9 \pi r^{2}-6 V=0
$$

Equation (1.15) can be solve using MATLAB software and we have non-zero critical value $r=6.9186$ for $V=$ $3000, r=8.2361$ for $V=5000, r=10.4236$ for $V=10000, r=11.9583$ for $V=15000$ and $r=13.1802$ for $V=20000$

Since $C^{\prime \prime}(r)>0$ for all above values of $r$ and $V$ then by second derivative test the cost of material equation (1.14) is minimum.

\subsection{Optimization of frustum of cone shape}

The volume of frustum of cone is

And the total surface area is

$$
V=\frac{1}{3} \pi h\left(R^{2}+R r+r^{2}\right)
$$

$$
S=\pi l(R+r)+\pi\left(R^{2}+r^{2}\right)=\pi\left[\sqrt{h^{2}+(R-r)^{2}}(R+r)+\left(R^{2}+r^{2}\right)\right]
$$

Where, $R$ and $r$ is upper and lower radius respectively, $h$ is the height and $l$ is slant height.

For this purpose we fix the base of the frustum and we will optimize the height and upper radius. The cost of material using the same material and sticking fragments is

$$
C=\pi(0.05)\left[\sqrt{\frac{9 V^{2}}{\pi^{2}\left(R^{2}+R r+r^{2}\right)^{2}}+(R-r)^{2}}(R+r)+R^{2}+r^{2}\right]
$$

To find the optimal value of cost we need to solve the following non-linear system of equation

$$
\left\{\begin{array}{c}
(R+r)\left[\frac{\pi^{3}(R-r)\left(R^{2}+R r+r^{2}\right)^{3}-9 V^{2}(2 R+r)}{\pi^{2}\left(R^{2}+R r+r^{2}\right)^{2}}\right]+\frac{9 V^{2}+\pi^{2}(R-r)^{2}\left(R^{2}+R r+r^{2}\right)^{2}}{\pi\left(R^{2}+R r+r^{2}\right)}+ \\
2 R \sqrt{9 V^{2}+\pi^{2}(R-r)^{2}\left(R^{2}+R r+r^{2}\right)}=0 \\
(R+r)\left[\frac{\pi^{3}(r-R)\left(R^{2}+R r+r^{2}\right)^{3}-9 V^{2}(2 r+R)}{\pi^{2}\left(R^{2}+R r+r^{2}\right)^{2}}\right]+\frac{9 V^{2}+\pi^{2}(R-r)^{2}\left(R^{2}+R r+r^{2}\right)^{2}}{\pi\left(R^{2}+R r+r^{2}\right)}+ \\
2 r \sqrt{9 V^{2}+\pi^{2}(R-r)^{2}\left(R^{2}+R r+r^{2}\right)}=0
\end{array}\right.
$$

Equation (1.17) can be solve using MATLAB software and we have non-zero critical value $R=r=7.8159$ for $V=3000, R=r=9.2668$ for $V=5000, R=r=11.6754$ for $V=10000, R=$ $r=13.3650$ for $V=15000$ and $R=r=14.7101$ for $V=20000$

Clearly, by second derivative test the cost of material equation (1.16) is minimum. The cost of material using the same material and folding fragments is 


$$
C=\pi(0.05)\left[\sqrt{\frac{9 V^{2}}{\pi^{2}\left(R^{2}+R r+r^{2}\right)^{2}}+(R-r)^{2}}(R+r)+R^{2}+r^{2}+2.5 R\right]
$$

To find the optimal value of cost we need to solve the following non-linear system of equation

$$
\left\{\begin{array}{c}
(R+r)\left[\frac{\pi^{3}(R-r)\left(R^{2}+R r+r^{2}\right)^{3}-9 V^{2}(2 R+r)}{\pi^{2}\left(R^{2}+R r+r^{2}\right)^{2}}\right]+\frac{9 V^{2}+\pi^{2}(R-r)^{2}\left(R^{2}+R r+r^{2}\right)^{2}}{\pi\left(R^{2}+R r+r^{2}\right)}+ \\
2(R+1.25) \sqrt{9 V^{2}+\pi^{2}(R-r)^{2}\left(R^{2}+R r+r^{2}\right)}=0 \\
(R+r)\left[\frac{\pi^{3}(r-R)\left(R^{2}+R r+r^{2}\right)^{3}-9 V^{2}(2 r+R)}{\pi^{2}\left(R^{2}+R r+r^{2}\right)^{2}}\right]+\frac{9 V^{2}+\pi^{2}(R-r)^{2}\left(R^{2}+R r+r^{2}\right)^{2}}{\pi\left(R^{2}+R r+r^{2}\right)}+ \\
2 r \sqrt{9 V^{2}+\pi^{2}(R-r)^{2}\left(R^{2}+R r+r^{2}\right)}=0
\end{array}\right.
$$

Equation (1.19) can be solve using MATLAB software and we have non-zero critical value $R=7.0586, r=$ 8.1110 for $V=3000, R=8.5246, r=9.5555$ for $V=5000, R=10.9487, r=11.9576$ for $V=10000$, $R=12.6454, r=13.6441$; for $V=15000$ and $R=13.9948, r=14.9874$ for $V=20000$

Clearly, by second derivative test the cost of material equation (1.18) is minimum. The cost of material using different material and sticking fragments is

$$
C=\pi\left[\sqrt{\frac{9 V^{2}}{\pi^{2}\left(R^{2}+R r+r^{2}\right)^{2}}+(R-r)^{2}}(R+r)(0.05)+(0.06) R^{2}+(0.08) r^{2}\right]
$$

To find the optimal value of cost we need to solve the following non-linear system of equation

$$
\left\{\begin{array}{c}
(R+r)\left[\frac{\pi^{3}(R-r)\left(R^{2}+R r+r^{2}\right)^{3}-9 V^{2}(2 R+r)}{\pi^{2}\left(R^{2}+R r+r^{2}\right)^{2}}\right]+\frac{9 V^{2}+\pi^{2}(R-r)^{2}\left(R^{2}+R r+r^{2}\right)^{2}}{\pi\left(R^{2}+R r+r^{2}\right)}+ \\
2.4 R \sqrt{9 V^{2}+\pi^{2}(R-r)^{2}\left(R^{2}+R r+r^{2}\right)}=0 \\
(R+r)\left[\frac{\pi^{3}(r-R)\left(R^{2}+R r+r^{2}\right)^{3}-9 V^{2}(2 r+R)}{\pi^{2}\left(R^{2}+R r+r^{2}\right)^{2}}\right]+\frac{9 V^{2}+\pi^{2}(R-r)^{2}\left(R^{2}+R r+r^{2}\right)^{2}}{\pi\left(R^{2}+R r+r^{2}\right)}+ \\
2.4 r \sqrt{9 V^{2}+\pi^{2}(R-r)^{2}\left(R^{2}+R r+r^{2}\right)}=0
\end{array}\right.
$$

Equation (1.21) can be solve using MATLAB software and we have non-zero critical value $R=8.1144, r=$ 5.7346 for $V=3000, R=9.6207, r=6.7991$ for $V=5000, R=12.1214, r=8.5663$ for $V=10000$, $R=13.8755, r=9.8060$ for $V=15000$ and $R=15.2719, r=10.7929$ for $V=20000$

Clearly, by second derivative test the cost of material equation (1.20) is minimum. The cost of material using different material and folding fragments is

$$
C=\pi\left[\sqrt{\frac{9 V^{2}}{\pi^{2}\left(R^{2}+R r+r^{2}\right)^{2}}+(R-r)^{2}}(R+r)(0.05)+(0.06) R^{2}+(0.08) r^{2}+0.15 R\right]
$$

To find the optimal value of cost we need to solve the following non-linear system of equation

$$
\left\{\begin{array}{c}
(R+r)\left[\frac{\pi^{3}(R-r)\left(R^{2}+R r+r^{2}\right)^{3}-9 V^{2}(2 R+r)}{\pi^{2}\left(R^{2}+R r+r^{2}\right)^{2}}\right]+\frac{9 V^{2}+\pi^{2}(R-r)^{2}\left(R^{2}+R r+r^{2}\right)^{2}}{\pi\left(R^{2}+R r+r^{2}\right)}+ \\
(2.4 R+3) \sqrt{9 V^{2}+\pi^{2}(R-r)^{2}\left(R^{2}+R r+r^{2}\right)}=0 \\
(R+r)\left[\frac{\pi^{3}(r-R)\left(R^{2}+R r+r^{2}\right)^{3}-9 V^{2}(2 r+R)}{\pi^{2}\left(R^{2}+R r+r^{2}\right)^{2}}\right]+\frac{9 V^{2}+\pi^{2}(R-r)^{2}\left(R^{2}+R r+r^{2}\right)^{2}}{\pi\left(R^{2}+R r+r^{2}\right)}+ \\
(2.4 r+3) \sqrt{9 V^{2}+\pi^{2}(R-r)^{2}\left(R^{2}+R r+r^{2}\right)}=0
\end{array}\right.
$$

Equation (1.23) can be solve using MATLAB software and we have non-zero critical value $R=7.3879, r=$ 6.2343 for $V=3000, R=8.9063, r=7.2938$ for $V=5000, R=11.4194, \quad r=9.056$ for $V=10000$, $R=13.1793, r=10.2934$. for $V=15000$ and $R=14.5793, r=11.2789$ for $V=20000$

Therefore, by second derivative test the cost of material equation (1.22) is minimum.

\section{Comparison of material cost of product between mathematical optimization and traditional method}

There are few numbers of plastic industries manufacture thousands of product having different shape and size yearly mostly for domestic demands. The most popular shapes are cylindrical, rectangular, hemisphere on top of a cylinder and frustum of cone. The cylindrical and rectangular shape of product have a volume $500 \mathrm{c} . \mathrm{m}^{3}, 1000 \mathrm{c} . \mathrm{m}^{3}$, $2000 \mathrm{c} \cdot \mathrm{m}^{3}, 3000 \mathrm{c} \cdot \mathrm{m}^{3}$ and $5000 \mathrm{c} \cdot \mathrm{m}^{3}$. But the top of the product have two types of seal. One is folding the other is sticking fragments. The shape which has hemisphere on top of a cylinder and frustum of cone have a volume 3000c.m $\mathrm{m}^{3}, 5000 \mathrm{c} . \mathrm{m}^{3}, 10000 \mathrm{c} . \mathrm{m}^{3}, 15000 \mathrm{c} . \mathrm{m}^{3}$ and 20000c.m ${ }^{3}$.

In appendix 1 and appendix 2 the existing products mostly preferable using the same material demand and cost are stated. In appendix 3 and appendix 4 the existing products mostly preferable using different material demand and cost are stated.

Based on Ethiopian manufacturing survey study most raw materials used in the subsector are available domestically. The production of processed products therefore to a large extent depends on the seasonality of weather, which largely determines the quality of the materials available. Our estimation approach focused on 
identifying and quantifying the loss of cost associated with the selection of dimension of products. Average material cost estimates per square centimeter were calculated and taken. To see the difference of cost between traditional and mathematical optimization selection of dimension products it is better considering only material cost. Based on our observation on training, most of industrial college teachers as well as industries works have no understanding dimension selection of product has an effect on material cost.

The survey has also tried to identify major challenges food and beverage products producer faces while selling products in the domestic and foreign markets. Top on the list are high cost of production compared to imported goods, low tariff protection, Insurgent of illegal goods and lack of access to market as major domestic market challenges. In similar fashion high cost of production compared to other competitor, lack of knowledge about foreign market trends, low capacity to produce in bulk and inability to keep product standards were most frequently mentioned foreign market challenges by respondent companies.

We consider 40 different mostly popular products based on their size and thickness. To show the effect 15,000 pieces for each size and shapes are taken. The number of products are increasing rapidly because personal and different manufactures demand are also increase. Since, our objective to show the difference of material cost between mathematical optimization and traditional methods due to choice of dimension of products.

The result of material cost for cylindrical shape using mathematical optimization and traditional existing product dimension selection are presented graphically as follows.

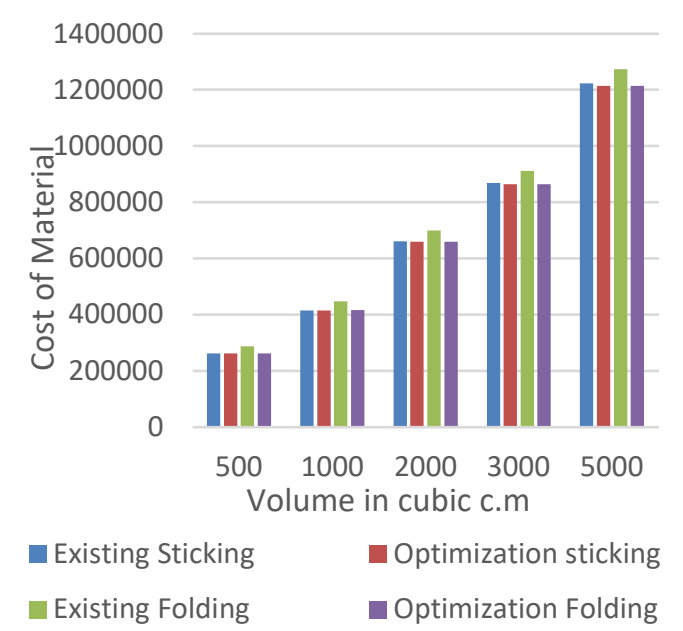

Figure1: The same thickness material cost for Cylindrical shape

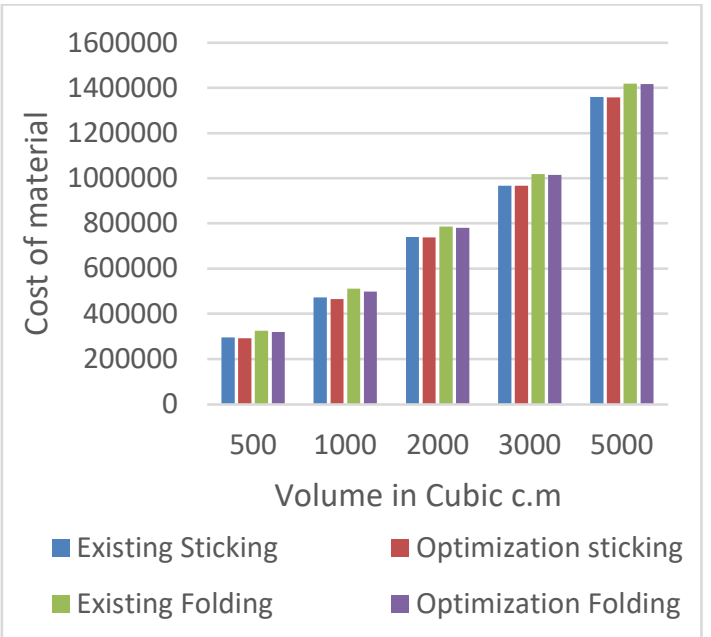

Figure2: Different thickness material cost for cylindrical shape

The material cost difference is vary from $34.35 \mathrm{ETB}$ to $58353.98 \mathrm{ETB}$. The minimum difference is using the same material by sticking top fragments for 500 cubic centimeter volume. The maximum difference is using the same material by folding top fragments for 5000 cubic centimeter volume.

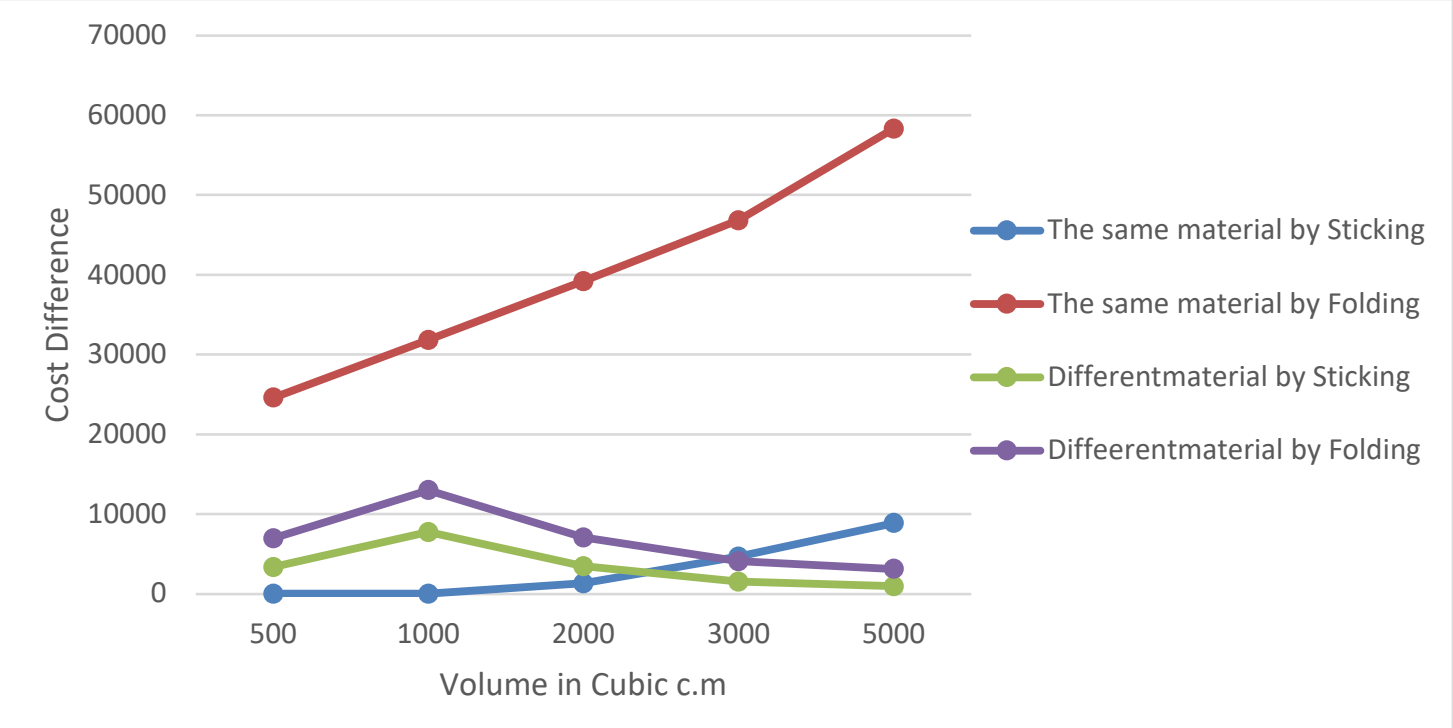

Figure3: Material cost difference between mathematical optimization and traditional method dimension selection of cylindrical shape products 
The result of material cost for rectangular shape using mathematical optimization and traditional existing product dimension selection are presented graphically as follows.

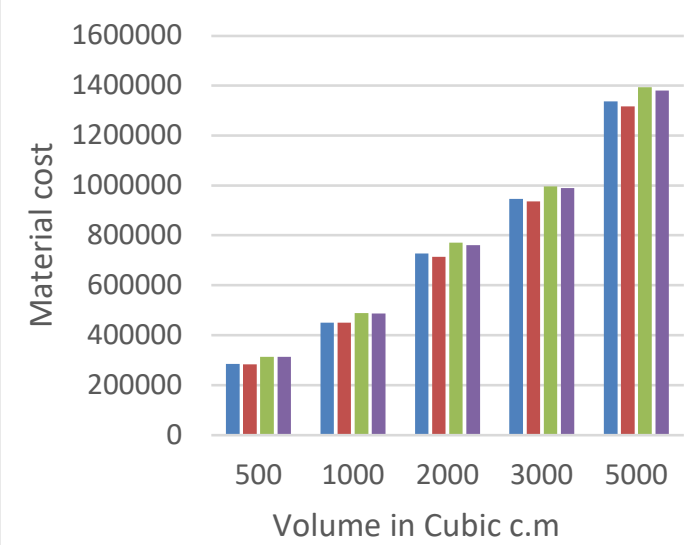

Existing Sticking

- Optimization sticking

Existing Folding

Figure 4: The same thickness material cost for rectangular shape

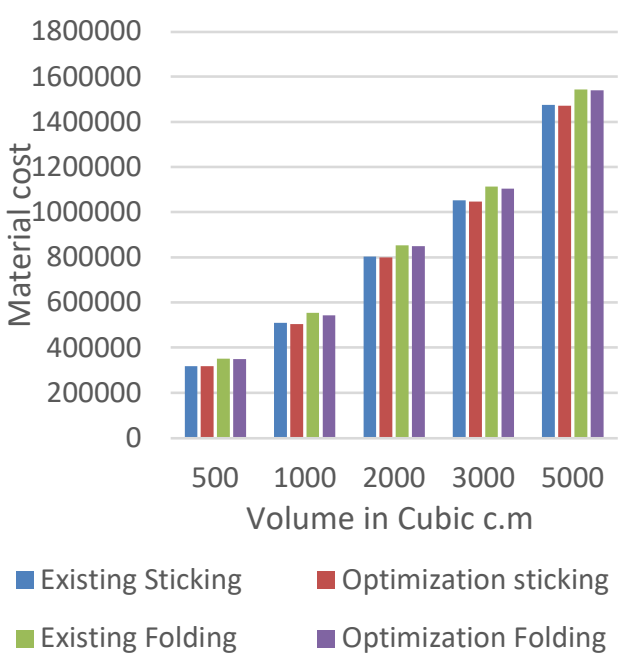

Figure 5: Different thickness material cost for rectangular shape

The material cost difference is vary 0 - 20951.76ETB. There is no cost difference using the same material by sticking top fragments for 1000 cubic centimeter volume. The maximum difference is using the same material by sticking top fragments for 5000 cubic centimeter volume.

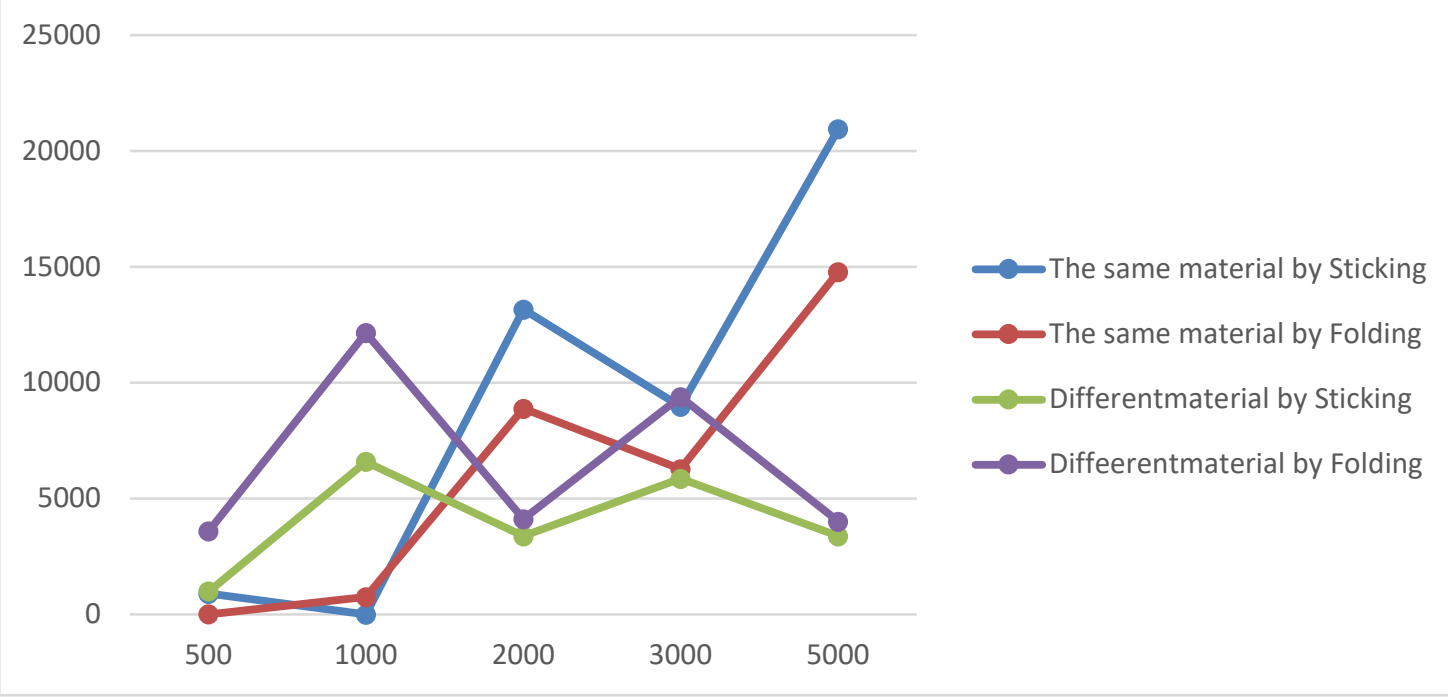

Figure 6: Material cost difference between mathematical optimization and traditional method Dimension selection of rectangular shape products

The material cost for hemispherical on the top of cylinder shape using mathematical optimization and traditional existing product dimension selection are presented graphically as follows. 


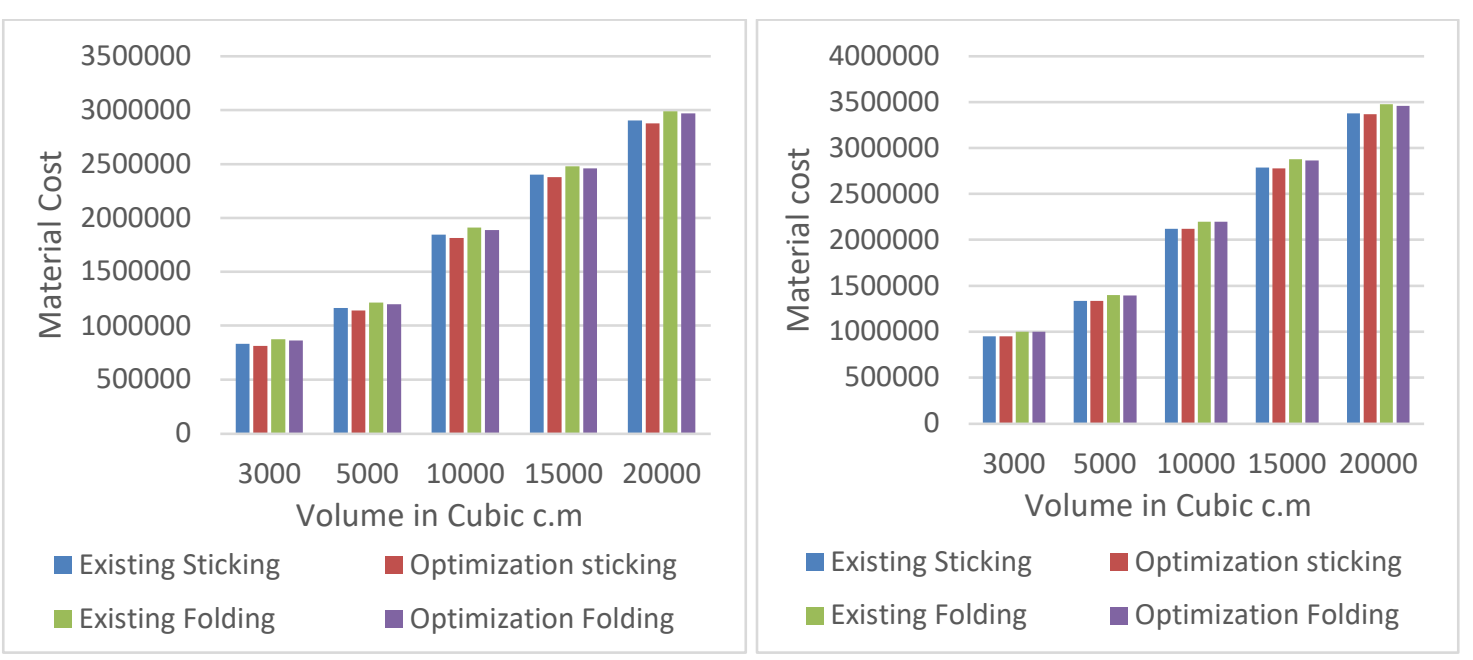

Figure 7: The same thickness material cost for hemispherical on top of cylinder
Figure 8: Different thickness material cost for hemispherical on top pf cylinder

The material cost difference is vary 2.53 - 32199.05ETB. The minimum cost difference exists on different material by sticking top fragments for 3000 cubic centimeter volume. The maximum cost difference exists on the same material by sticking top fragments for 10000 cubic centimeter volume.

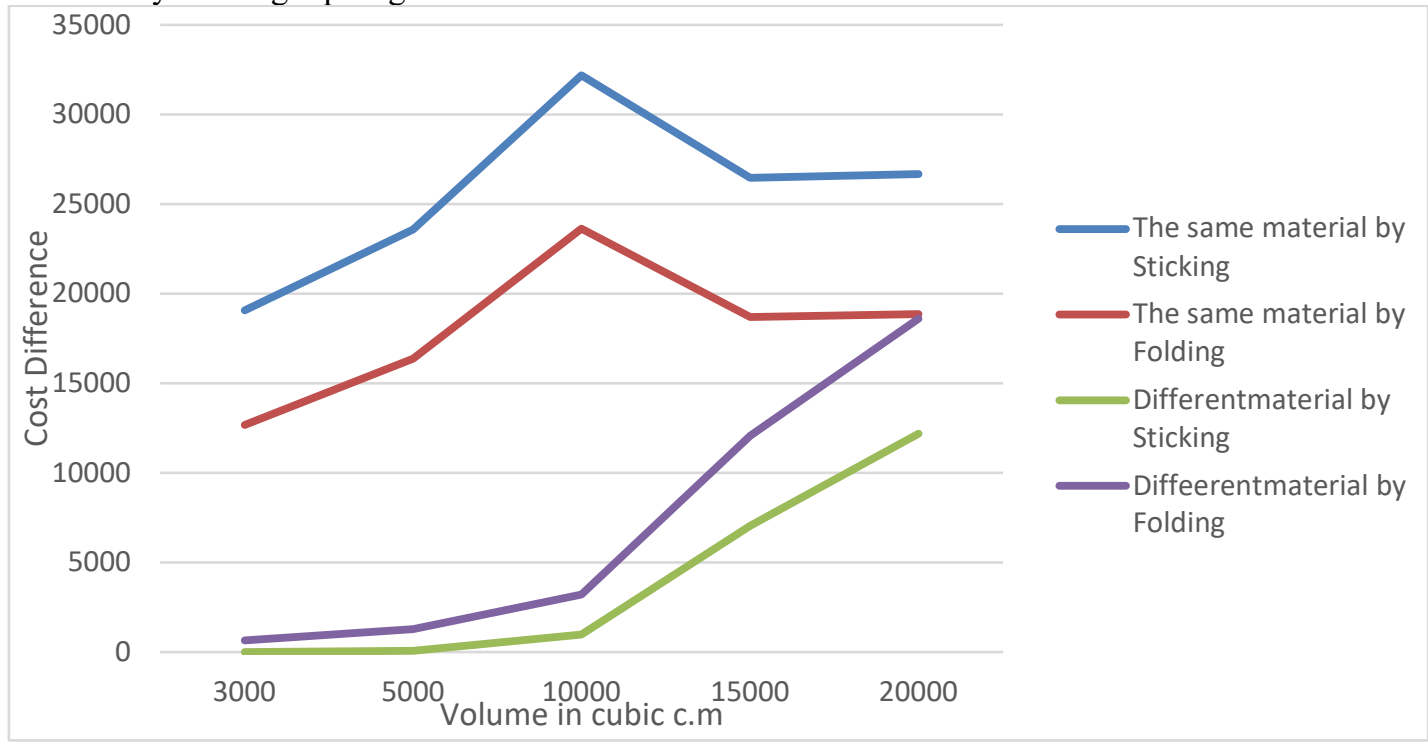

Figure 9: Material cost difference between mathematical method and traditional method for hemispherical On top of cylinder

The material cost for frustum of cone shape using mathematical optimization and traditional existing product dimension selection are presented graphically as follows. 


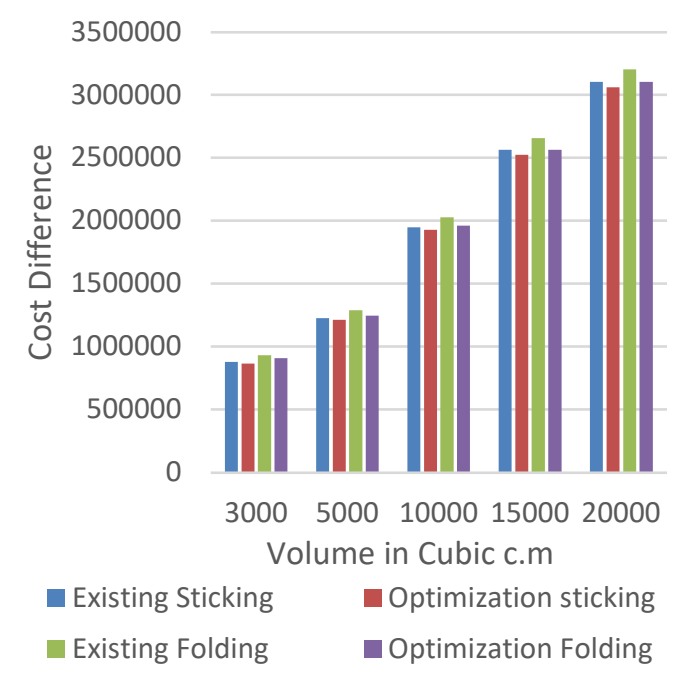

Figure 10: The same thickness material cost for frustum of cone

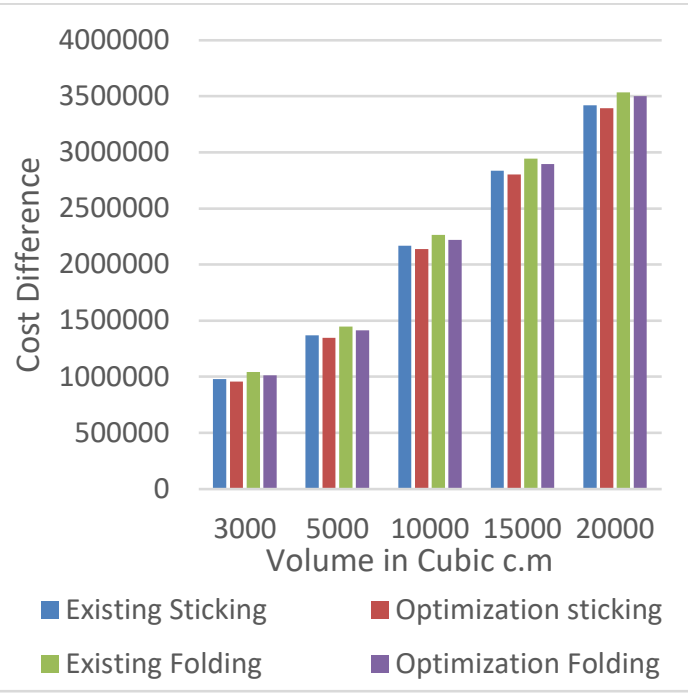

Figure 11: Different thickness material cost for Frustum of cone

The material cost difference is vary 12179.73 - 99947.33ETB. The minimum cost difference exists on the same material by sticking top fragments for 5000 cubic centimeter volume. The maximum cost difference exists on the same material by folding top fragments for 20000 cubic centimeter volume.

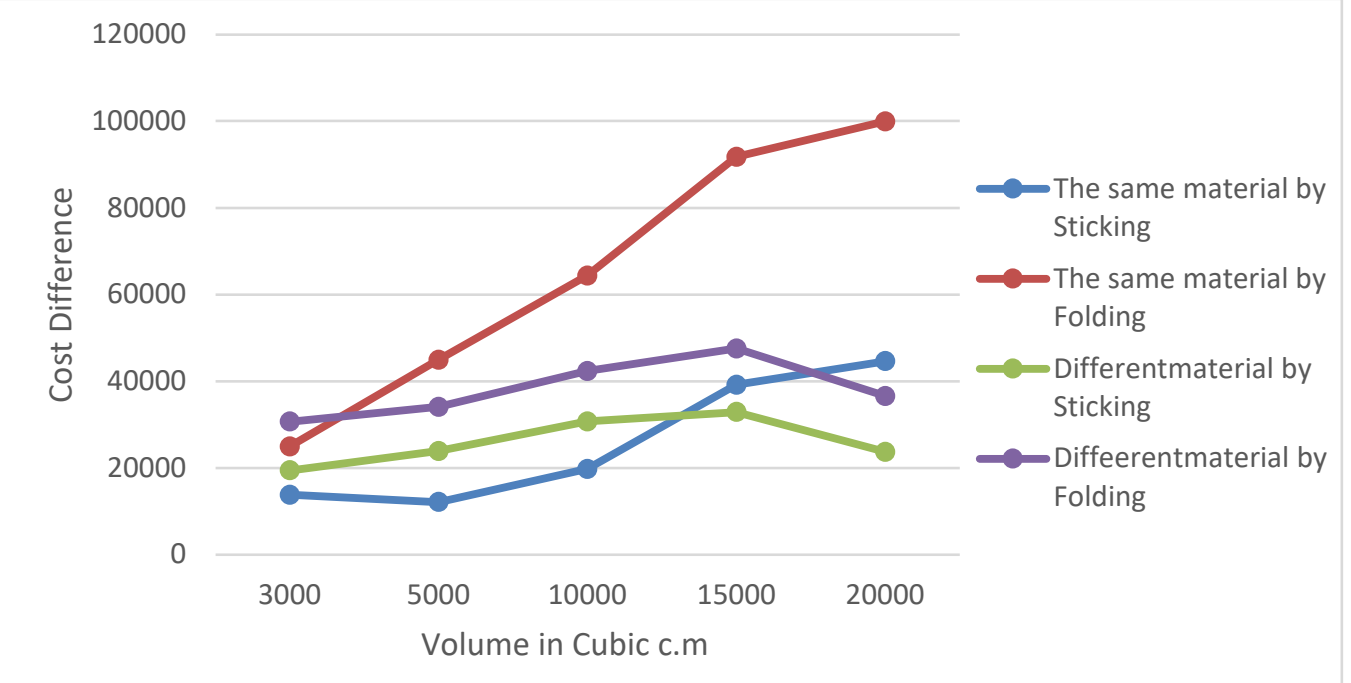

Figure 12: Material cost difference between mathematical method and traditional method for Frustum of cone

\section{Discussion}

Figure 1 and Figure 2 in the above shows that the material cost of cylindrical shape products presented both mathematical optimization and existing traditional selection of dimension. As shown in figure 3 product dimension selection is crucial for minimize cost. Small size products 500 and 1000 cubic centimeter using the same material by sticking fragments cost difference is low. But for all size of product using the same material by folding fragments is large compared to all other products. Figure 4 and Figure 5 shows that the material cost of rectangular shape products presented both mathematical optimization and existing traditional selection of dimension. From all 40 selected products only 1000 cubic centimeter volume using the same material sticking fragments is best choice of dimension as shown in figure 6 .

Figure 7 and Figure 8 shows that the material cost of hemispherical on the top of cylinder shape products presented both mathematical optimization and existing traditional selection of dimension. In this shape the minimum cost difference was exist when we used different material by sticking fragments and the maximum cost difference was exist when we used the same material by sticking fragments as shown in figure 9 . The second minimum material cost difference exist on products using different material by folding fragments. Similarly, Figure 10 and Figure 11 shows that the material cost of frustum of cone shape products presented both mathematical optimization and existing traditional selection of dimension. As shown in figure 12, the minimum 
cost difference was exist on the size 3000,5000 and 10000 cubic centimeter using the same material by sticking fragments. The maximum material cost difference was exist when industries used the same material by folding fragments for all size except at volume 3000 cubic centimeters. Furthermore, the maximum material cost difference throughout all selected 40 products exist on volume 20000 cubic centimeters using the same material by folding fragments.

\section{Conclusion}

The study has been clearly shows that product dimension selection is crucial for minimize the material cost. For all selected industrial products the optimal dimension of products are presented by solving nonlinear system of equation. Mathematical optimization manufacturing is not only has implications for the costs of production, but also the utilization of the final product.

Selection of dimension of products traditionally in Ethiopia has large number of material wastage and exposed for unnecessary material cost. The material cost for a single product range is up to $99947.33 \mathrm{ETB}$, using the same material cost difference is $964338.75 \mathrm{ETB}$ and different material cost difference is 483053.41ETB. Hence, from all selected 40 different size and shape products the total material cost difference is $1447392.16 \mathrm{ETB}$.

\section{References}

1. Central Statistical Agency (CSA), Ethiopia, various issues. Survey on Large and Medium Scale Manufacturing Industries.

2. DAB Development Research and training PLC, Ethiopian Manufacturing survey analysis, 2014, Addis Ababa

3. UNDP, Case study of Growing Manufacturing Industry in Ethiopia

4. Tekeba Eshetie, Ethiopia's Manufacturing Industry Opportunities, Challenges and Way Forward (A Sectoral Overview), Crimson Publishers, 2018

5. Central Statistical Agency(CSA), Ethiopia - Statistical Abstract - 2013/2014, Addis Ababa, March 2015

6. The Embassy of Japan in Ethiopia, A Series of Studies on Industries in Ethiopia, 2008

7. Mulu Gebreeyesus, Industrial policy and development in Ethiopia Evolution and present experimentation,

8. Ministry of Industry, Summary Feasibility Studies for Selected Sub sectors, 2018

9. UNECA, 2016. Transformative Industrial Policy, United Nations Economic Commission for Africa, Addis Ababa.

10. UNECA, 2015. Industrializing through trade, Economic Report on Africa, United Nations Economic Commission for Africa, Addis Ababa

11. Ministry of Finance and Economic Development (MOFED), Growth and Transformation Plan (GTP) Annual Progress Report for F.Y 2012/2013, Addis Ababa, February 2014

12. JAMES C. ROBINSON, 2004. Introduction to Ordinary Differential Equations. Cambridge University Press, New York.

13. John H. Mathews and Kurtis K. Fink, 2004. Numerical Methods Using MATLAB. Prentice-Hall Inc., New Jersey.

\section{Appendices}

Table 1. The material demand and cost of material using the same thickness material by sticking fragments per product

\begin{tabular}{|c|c|c|c|c|c|c|c|c|}
\hline \multirow[b]{2}{*}{$\begin{array}{l}\text { Volume } \\
\left(\text { c. } m^{3}\right)\end{array}$} & \multicolumn{2}{|c|}{ Cylindrical shape } & \multicolumn{2}{|c|}{ Rectangular shape } & \multicolumn{2}{|c|}{$\begin{array}{l}\text { Hemispherical on top } \\
\text { of Cylindrical Shape }\end{array}$} & \multicolumn{2}{|c|}{ Frustum of cone } \\
\hline & 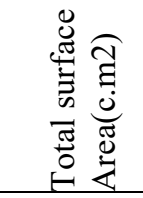 & 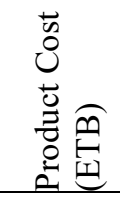 & 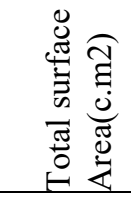 & 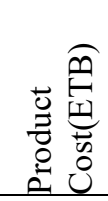 & 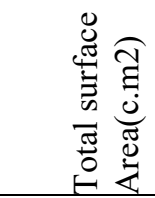 & 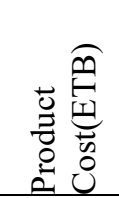 & 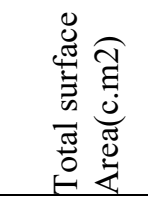 & 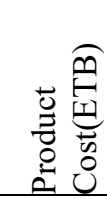 \\
\hline 500 & 348.78 & 17.44 & 379.2 & 18.96 & 2 & - & 2 & $=2$ \\
\hline 1000 & 553.61 & 27.68 & 600 & 30 & - & - & - & \\
\hline 2000 & 880.49 & 44.02 & 970 & 48.5 & - & - & - & \\
\hline 3000 & 1157.73 & 57.89 & 1260.18 & 63.01 & $\overline{1} 109.02$ & $\overline{5} 5.45$ & $\overline{1} 169.93$ & $\overline{5} 8.5$ \\
\hline 5000 & 1630.52 & 81.53 & 1782.65 & 89.13 & 1554.68 & 77.73 & 1634.92 & 81.75 \\
\hline 10000 & & - & 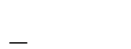 & - & 2460.92 & 123.05 & 2595.90 & 129.79 \\
\hline 15000 & - & - & & & 3203.73 & 160.19 & 3419.27 & 170.96 \\
\hline 20000 & & & & & 3873.88 & 193.69 & 4138.35 & 206.92 \\
\hline Total & 4222.35 & 211.12 & 4612.83 & 230.64 & 12202.23 & 610.11 & 12958.37 & 647.92 \\
\hline
\end{tabular}


Table 2. The material demand and cost of material using the same thickness material by folding fragments per product

\begin{tabular}{|c|c|c|c|c|c|c|c|c|}
\hline \multirow[b]{2}{*}{$\begin{array}{l}\text { Volume } \\
\left(\text { c. } m^{3}\right)\end{array}$} & \multicolumn{2}{|c|}{ Cylindrical shape } & \multicolumn{2}{|c|}{ Rectangular shape } & \multicolumn{2}{|c|}{$\begin{array}{l}\text { Hemispherical on top } \\
\text { of Cylindrical Shape }\end{array}$} & \multicolumn{2}{|c|}{ Frustum of cone } \\
\hline & 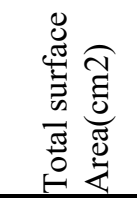 & 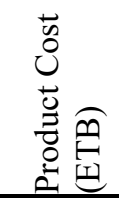 & 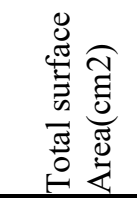 & 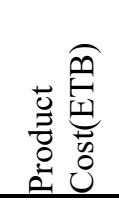 & 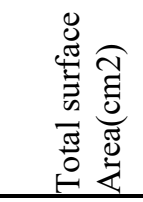 & 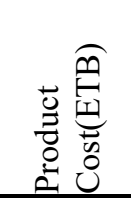 & 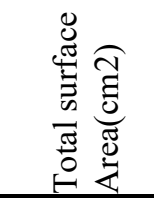 & 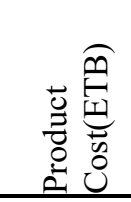 \\
\hline 500 & 382.32 & 19.12 & 416.67 & 20.83 & - & - & - & - \\
\hline 1000 & 596.81 & 29.84 & 650 & 32.5 & - & - & - & - \\
\hline 2000 & 931.78 & 46.59 & 1026.25 & 51.31 & & & & \\
\hline 3000 & 1214.75 & 60.74 & 1327.5 & 66.38 & $\overline{1} 164.78$ & $\overline{5} 8.24$ & $\overline{1} 243.37$ & $\overline{6} 2.168$ \\
\hline 5000 & 1697.28 & 84.86 & 1858.6 & 92.93 & 1621.44 & 81.07 & 1719.12 & 85.956 \\
\hline 10000 & - & $-1+2$ & & - & 2545.98 & 127.3 & 2701.30 & 135.07 \\
\hline 15000 & - & - & & - & 3303.95 & 165.2 & 3541.87 & 177.09 \\
\hline 20000 & & & & & 3985.25 & 199.26 & 4269.90 & 213.5 \\
\hline Total & 4822.94 & 222.03 & 4862.83 & 243.14 & 12621.4 & 631.07 & 13475.56 & 673.784 \\
\hline
\end{tabular}

Table 3. The material demand and cost of material using the different thickness material by sticking fragments per product

\begin{tabular}{|c|c|c|c|c|c|c|c|c|}
\hline \multirow[b]{2}{*}{$\begin{array}{l}\text { Volume } \\
\left(\text { c. } m^{3}\right)\end{array}$} & \multicolumn{2}{|c|}{ Cylindrical shape } & \multicolumn{2}{|c|}{ Rectangular shape } & \multicolumn{2}{|c|}{$\begin{array}{l}\text { Hemispherical on top } \\
\text { of Cylindrical Shape }\end{array}$} & \multicolumn{2}{|c|}{ Frustum of cone } \\
\hline & 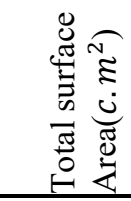 & 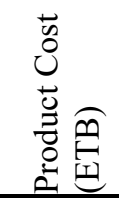 & 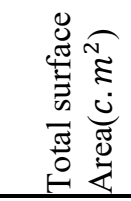 & 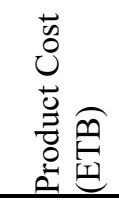 & 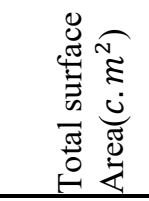 & 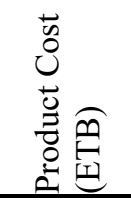 & 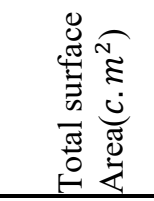 & 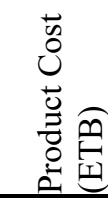 \\
\hline 500 & 348.78 & 19.73 & 379.2 & 21.21 & 2 & -6 & $x_{2}$ & - \\
\hline 1000 & 553.61 & 31.48 & 600 & 34 & - & - & - & - \\
\hline 2000 & 880.49 & 49.38 & 970 & 53.5 & & & & \\
\hline 3000 & 1157.73 & 64.51 & 1260.18 & 70.21 & $\overline{1} 109.02$ & $\overline{6} 3.37$ & $\overline{1} 169.93$ & $\overline{6} 5.19$ \\
\hline 5000 & 1630.52 & 90.61 & 1782.65 & 98.37 & 1554.68 & 89.08 & 1634.92 & 91.40 \\
\hline 10000 & $\ldots$ & - & te & - & 2460.92 & 141.47 & 2595.90 & 144.62 \\
\hline 15000 & - & - & - & - & 3203.73 & 185.76 & 3419.27 & 189.01 \\
\hline 20000 & & & & & 3873.88 & 225.28 & 4138.35 & 227.89 \\
\hline Total & 4222.35 & 235.98 & 4612.83 & 256.08 & 12202.23 & 704.96 & 12958.37 & 718.11 \\
\hline
\end{tabular}

Table 4: The material demand and cost of material using different thickness material by folding fragments per product

\begin{tabular}{|c|c|c|c|c|c|c|c|c|}
\hline \multirow[b]{2}{*}{$\begin{array}{l}\text { Volume } \\
\left(\text { c. } m^{3}\right)\end{array}$} & \multicolumn{2}{|c|}{ Cylindrical shape } & \multicolumn{2}{|c|}{ Rectangular shape } & \multicolumn{2}{|c|}{$\begin{array}{l}\text { Hemispherical on top } \\
\text { of Cylindrical Shape }\end{array}$} & \multicolumn{2}{|c|}{ Frustum of cone } \\
\hline & 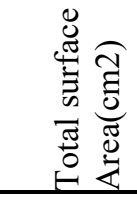 & 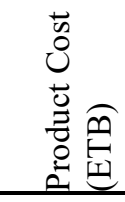 & 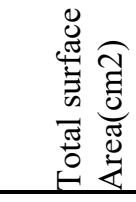 & 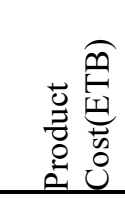 & 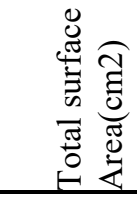 & 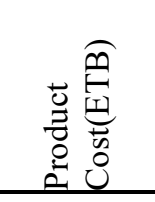 & 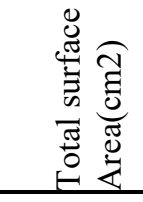 & 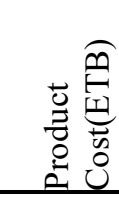 \\
\hline 500 & 382.32 & 21.74 & 416.67 & 23.46 & - & - & - & - \\
\hline 1000 & 596.81 & 34.07 & 650 & 37 & - & 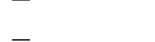 & - & _ \\
\hline 2000 & 931.78 & 52.46 & 1026.25 & 56.88 & & & - & - \\
\hline 3000 & 1214.75 & 67.93 & 1327.5 & 74.25 & $\overline{1} 164.78$ & $\overline{6} 6.72$ & $\overline{1} 243.37$ & $\overline{6} 9.59$ \\
\hline 5000 & 1697.28 & 94.61 & 1858.6 & 102.93 & 1621.44 & 93.09 & 1719.12 & 96.45 \\
\hline 10000 & 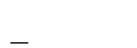 & - & 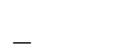 & 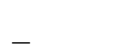 & 2545.98 & 146.57 & 2701.30 & 150.94 \\
\hline 15000 & - & - & & & 3303.95 & 191.77 & 3541.87 & 196.37 \\
\hline 20000 & & & & & 3985.25 & 231.96 & 4269.90 & 235.79 \\
\hline Total & 4822.94 & 270.81 & 4862.35 & 294.52 & 12621.4 & 730.11 & 9205.66 & 513.35 \\
\hline
\end{tabular}

